\title{
Fundamental considerations for multiwavelength photoacoustic molecular imaging
}

Roger J. Zemp, Li Li, Lihong V. Wang

Roger J. Zemp, Li Li, Lihong V. Wang, "Fundamental considerations for multiwavelength photoacoustic molecular imaging," Proc. SPIE 6086, Photons Plus Ultrasound: Imaging and Sensing 2006: The Seventh Conference on Biomedical Thermoacoustics, Optoacoustics, and Acousto-optics, 60861L (6 March 2006); doi: 10.1117/12.646392

SPIE. Event: SPIE BiOS, 2006, San Jose, California, United States 


\title{
Fundamental Considerations for Multiwavelength Photoacoustic Molecular Imaging
}

\author{
Roger J. Zemp, Li Li, Lihong V. Wang ${ }^{*}$ \\ Optical Imaging Laboratory, Department of Biomedical Engineering, \\ Texas A\&M University 77843-3120 \\ *1wang@tamu.edu
}

\begin{abstract}
Photoacoustic technology offers great promise for molecular imaging in vivo since it offers significant penetration, and optical contrast with ultrasonic spatial resolution. In this article we examine fundamental technical issues impacting capabilities of photoacoustic tomography for molecular imaging. First we examine how reconstructed photoacoustic tomography images are related to true absorber distributions by studying the modulation transfer function of a circular scanning tomographic system employing a modified filtered backprojection algorithm. We then study factors influencing quantitative estimation by developing a forward model of photoacoustic signal generation, and show conditions for which the system of equations can be inverted. Errors in the estimated optical fluence are shown to be a source of bias in estimates of molecular agent concentration. Finally we discuss noise propagation through the matrix inversion procedure and discuss implications for molecular imaging sensitivity and system design.
\end{abstract}

\section{Introduction}

Molecular imaging is a new paradigm that is gaining considerable momentum and is considered by many as the future of medical imaging ${ }^{1,2}$. Biophotonic imaging is already playing an important role in this movement ${ }^{3,4}$, however, key challenges still remain including the highly scattering nature of light in biological tissues. These challenges, however, are worth confronting. Optical contrast is a tremendously significant parameter considering the vast toolset of biotechnology leveraging optical contrast agents, not to mention exceptional endogenous tissue contrast. Regarding challenges due to scattering, photoacoustic tomography may come to the rescue. In photoacoustic imaging, short pulses of laser light irradiate tissue and are converted into acoustic energy via a thermoelastic expansion and the ultrasonic signals are detected at various spatial positions and reconstructed to form images representative of optical absorption in tissue $^{5}$. Light can be scattered over a large volume but the spatial resolution is due to ultrasonic detection. Multiple wavelengths of light can be used to probe the tissue and by using known spectral absorption curves of dominant absorbers, blood oxygenation and the distribution of molecular imaging agents can be extracted ${ }^{6,7,8}$. There are, however, fundamental issues and tradeoffs that impact the success of this strategy. These issues are the subject of this paper. In particular, this article will address the basic principles related to the capabilities of photoacoustic tomography for molecular imaging.

\section{Point-Spread and Modulation Transfer Functions}

The first question that will be addressed is how reconstructed photoacoustic images are related to the true absorption distributions. To understand this question it is necessary to understand the point-spread function (PSF) or modulation transfer function (MTF) of the tomographic imaging system. The PSF is the image formed due to a point absorber, and the MTF is the normalized magnitude of the Fourier Transform of the PSF.

For an infinite cylinder, enclosed sphere or planar array of detectors with infinite bandwidth, M. Xu et al. ${ }^{5}$ developed an exact reconstruction algorithm, similar to the filtered backprojection algorithm commonly used in X-ray computed tomography, but adapted for coherent time-domain signals. In our experiments, a single element bandlimited transducer is scanned in a circular geometry, and the detector array is in effect a ring rather than a higher dimensional detector 
surface. Y. Xu et al. proposed an approximate reconstruction algorithm for circular scanning assuming a large detector radius. We build on past efforts to evaluate the modulation transfer function for the circular scanning system with a modified filtered backprojection algorithm. The starting point for this analysis is the frequency domain filtered backprojection reconstruction formula from [5].

$$
A(\mathbf{r})=\int_{S_{0}} d s \frac{1}{2 \pi} \int_{-\infty}^{\infty} d k \tilde{p}\left(\mathbf{x}_{0}, k\right)(-i k) \frac{\exp \left(-i k\left|\mathbf{x}_{0}-\mathbf{r}\right|\right)}{\left|\mathbf{x}_{0}-\mathbf{r}\right|}
$$

where $A$ is the reconstructed image representative of optical absorption, $\mathbf{r}$ is the image space coordinate, $\widetilde{p}$ is the temporal Fourier transform of the pressure signal, $k$ is the acoustic wavenumber $2 \pi / \lambda$ ( $\lambda$ is the acoustic wavelength), and $\mathbf{x}_{0}$ is the object space coordinate. Other multiplicative constants are neglected for convenience. Fig. 1 shows the geometry of the coordinates

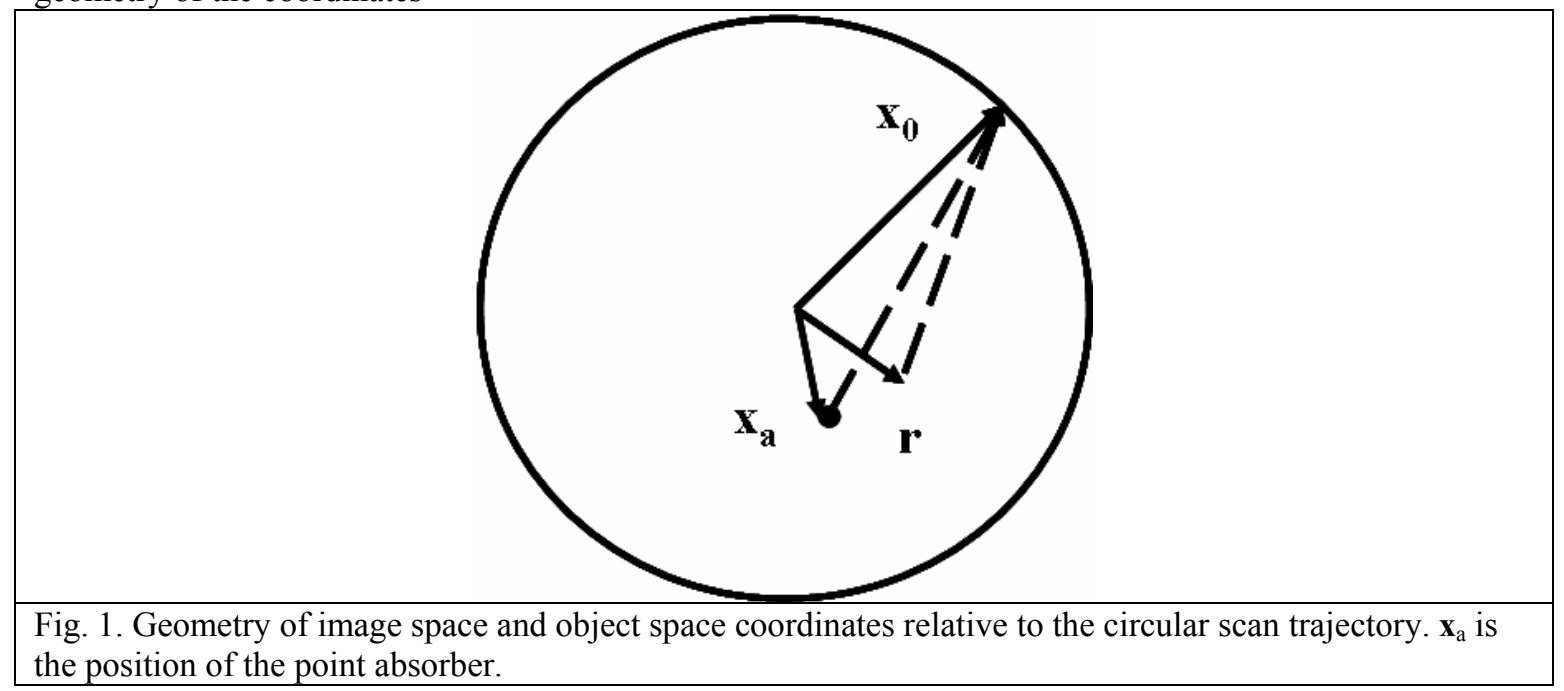

The point-spread function is produced by assuming substituting the pressure distribution from an in-plane point absorber at object space coordinate $\mathbf{x}_{\mathrm{a}}$ :

$\widetilde{p}\left(\mathbf{x}_{0}, k\right)=-i k \frac{\exp \left(i k\left|\mathbf{x}_{a}-\mathbf{x}_{0}\right|\right)}{4 \pi\left|\mathbf{x}_{a}-\mathbf{x}_{0}\right|} H_{b}(k)$

where we have included a free-space Green's function, a factor of $i k$ to convert velocity potential to pressure. Here $H_{\mathrm{b}}$ is the temporal frequency response of the transducer in terms of the argument $k=\omega / c$, where $\omega$ is angular frequency, and $c$ is the acoustic speed of sound. Other multiplicative constants are neglected for simplicity. When substituting this into (1), we obtain an expression for the point-spread function

$$
\operatorname{psf}\left(\mathbf{r} \mid \mathbf{x}_{a}\right)=\int_{S_{0}} d s \int_{-\infty}^{\infty} d k \frac{\exp \left[i k\left(\left|\mathbf{x}_{0}-\mathbf{x}_{a}\right|-\left|\mathbf{x}_{0}-\mathbf{r}\right|\right)\right]}{\left|\mathbf{x}_{0}-\mathbf{x}_{a} \| \mathbf{x}_{0}-\mathbf{r}\right|} k^{2} H_{b}(k) .
$$

At this point it is necessary to make some approximations. Specifically we assume that (1) the scan radius is much greater than the absorber distance to the scan center and (2) that the image space points of interest are close to the absorber compared to the scan radius. With these approximations the denominator factors are slowly varying effectively constant over the circumferential integration. The numerator phase factor is approximated as $\left|\mathbf{x}_{0}-\mathbf{x}_{a}\right|-\left|\mathbf{x}_{0}-\mathbf{r}\right| \approx R \cos \theta$ where $R$ is the distance from the point absorber to the image space coordinate $\mathbf{r}$, and where $\theta$ is a polar angle coordinate, as seen in Fig. 2 . 


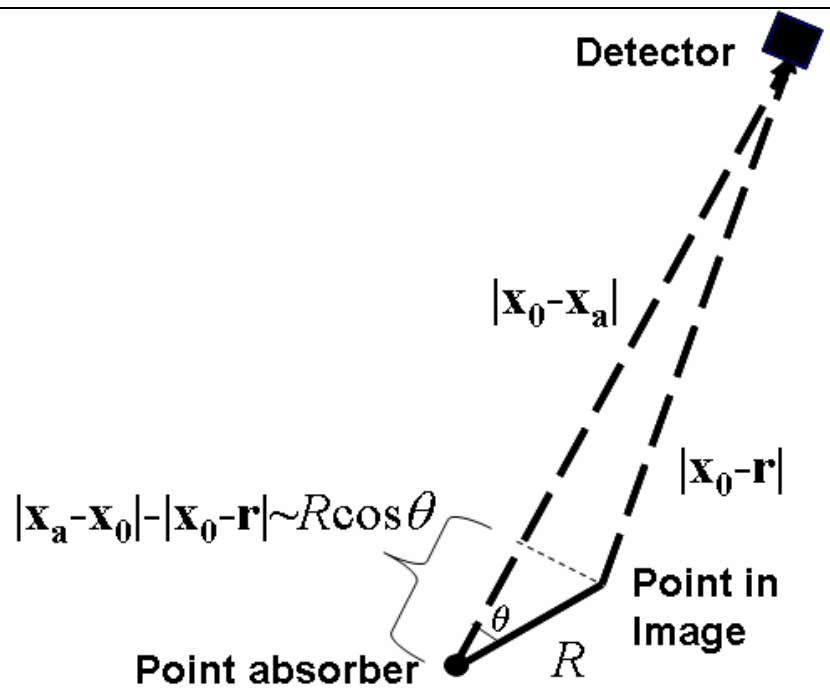

Fig. 2. Geometry showing point absorber and image space point in relation to the detector. For point absorber close to the detector scan and for large detector radii, the difference we may use the approximation $\left|\mathbf{x}_{0}-\mathbf{x}_{a}\right|-\left|\mathbf{x}_{0}-\mathbf{r}\right| \approx R \cos \theta$.

The line integral around the detector path then reduces to an integral over $\theta$.

$p s f(R) \approx \frac{1}{\left|\mathbf{x}_{0}\right|} \int_{-\infty}^{\infty} d k \int_{d \theta} d \theta(-i k)^{2} \exp (-i k R \cos \theta) H_{b}(k)$

$=\frac{1}{\left|\mathbf{x}_{0}\right|} \int_{-\infty}^{\infty}\left[-k H_{b}(k)\right] J_{0}(k R) k d k$,

where $J_{0}$ is a zero-th order Bessel function of the first kind. This looks very much like a Hankel transform except that the lower integration bound is $-\infty$ rather than 0 . Note that both $J_{0}$ and $k^{2}$ are even functions, hence odd parts of $H_{b}$ will integrate to zero, and the point-spread function can be written as

$\operatorname{psf}(R) \propto \frac{2}{\left|\mathbf{x}_{0}\right|} \int_{0}^{\infty}\left[-k H_{e}(k)\right] J_{0}(k R) k d k$

where $H_{\mathrm{e}}$ is the even part of $H_{b}$. This is simply the real part of $H_{b}$ considering that the temporal impulse response of the transducer is a real function, implying that $H_{b}(k)=H_{b}{ }^{*}(i k)$. The point-spread function is then approximated as the Hankel Transform of $k$ times the real part of the transducer frequency response. Here the acoustic wavevector $k$ may be interpreted as a radial spatial frequency $k=\sqrt{k_{x}^{2}+k_{y}^{2}}$ where $k_{\mathrm{x}}$ and $k_{y}$ are spatial frequencies conjugate to the image space scan-plane coordinates. The Modulation Transfer Function (MTF), defined as the normalized magnitude of the 2D Fourier Transform of the point-spread function is then given as

$\operatorname{MTF}\left(k_{x}, k_{y}\right) \propto \sqrt{k_{x}^{2}+k_{y}^{2}} \mid \operatorname{Re}\left\{H_{b}\left(\sqrt{k_{x}^{2}+k_{y}^{2}}\right)\right\}$.

Because transducers have a bandpass frequency response, this result tells us that the photoacoustic tomography system is sensitive to a radial band of spatial frequencies. This result is important for understanding how reconstructed photoacoustic tomography images are related to absorption distributions. It means that only certain absorber geometries (i.e. geometries with spatial frequency content in the passband of the MTF) will efficiently couple energy into the imaging system. For example, a large absorbing region such as a tumor may have low spatial frequency content, and the reconstructed image will only see the border where spatial frequencies are higher. Similarly, very fine capillary 
networks may have spatial frequency content beyond the system passband frequencies and may not be visible to the imaging system.

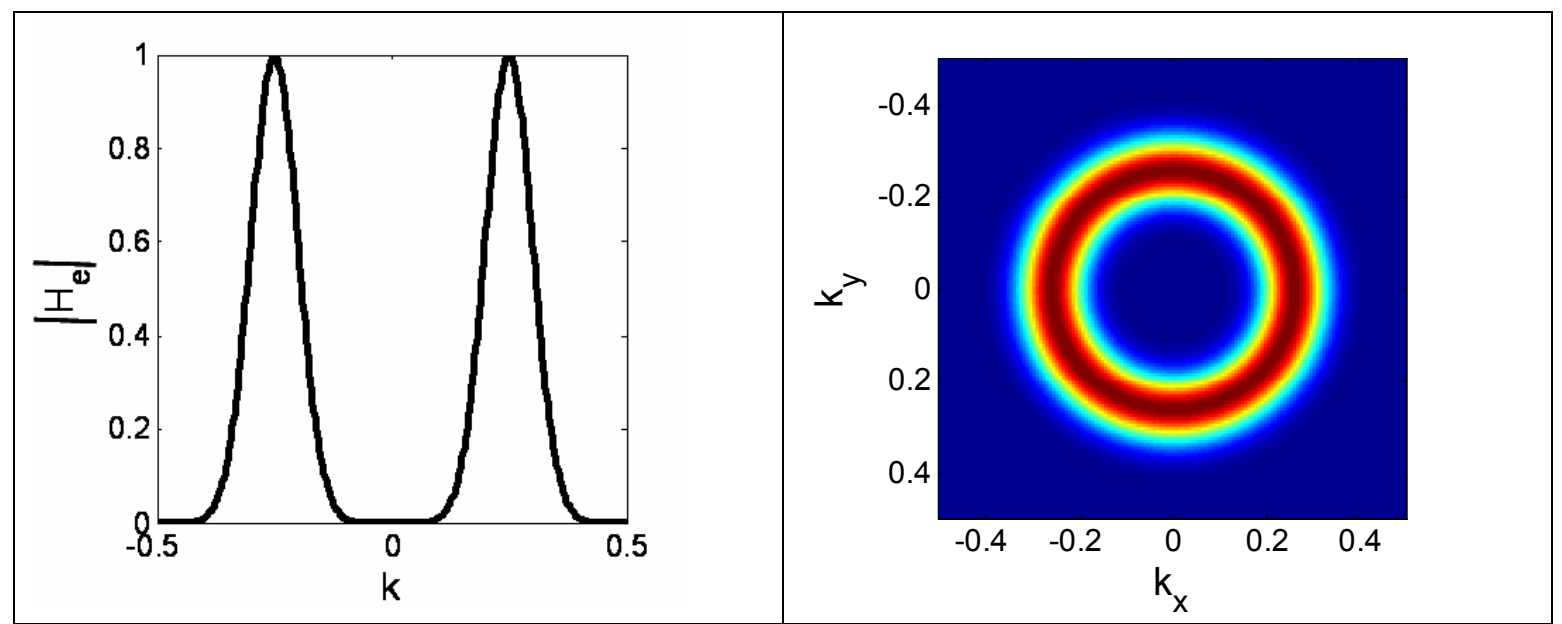

Fig. 3 (a) example real magnitude of transducer frequency response as a function of normalized frequency units. (b) Corresponding Modulation Transfer Function of the system.

\section{Multi-wavelength photoacoustic estimation of molecular agent distributions}

\subsection{Estimator Bias}

Consider now that we are interested in estimating the spatial concentration distribution of a molecular imaging agent in a living subject. Let's label the molecular imaging agent ' $b$ ', and assume that this, along with oxy-hemoglobin and deoxy-hemoglobin are the dominant absorbing species in tissue. The principle behind multi-wavelength estimation is to use various optical wavelengths to estimate concentration distributions of absorbing species with known molar extinction spectra by solving a set of (often overdetermined) equations. A reconstructed photoacoustic image $p_{\lambda_{j}}(\mathbf{r})$ for wavelength $\lambda_{\mathrm{j}}$ is given as:

$p_{j}(\mathbf{r})=\int h(\mathbf{x}, \mathbf{r}) \Phi_{j}(\mathbf{x}) \mu_{a, j}(\mathbf{x}) d \mathbf{x}$,

Here $h(\mathbf{x}, \mathbf{r})$ is the system impulse response that maps the object space $\mathbf{x}$ to the image space $\mathbf{r}, \Phi_{j}(\mathbf{x})$ is the optical fluence as a function of object space for wavelength $\lambda_{\mathrm{j}}$, and $\mu_{a, j}(\mathbf{x})$ is the total absorption at wavelength $\lambda_{\mathrm{j}}$. In matrix form this can be written as

$$
\left[\begin{array}{c}
p_{1}(\mathbf{r}) \\
p_{2}(\mathbf{r}) \\
\vdots \\
p_{N}(\mathbf{r})
\end{array}\right]=\int d \mathbf{x} h(\mathbf{x}, \mathbf{r})\left[\begin{array}{cccc}
\Phi_{1}(\mathbf{x}) & & & 0 \\
& \Phi_{2}(\mathbf{x}) & & \\
& & \ddots & \\
0 & & & \Phi_{N}(\mathbf{x})
\end{array}\right]\left[\begin{array}{ccc}
\varepsilon_{H b, \lambda_{1}} & \varepsilon_{H b O_{2}, \lambda_{1}} & \varepsilon_{b, \lambda_{1}} \\
\varepsilon_{H b, \lambda_{2}} & \varepsilon_{H b O_{2}, \lambda_{2}} & \varepsilon_{b, \lambda_{2}} \\
\vdots & \vdots & \vdots \\
\varepsilon_{H b, \lambda_{N}} & \varepsilon_{H b O_{2}, \lambda_{N}} & \varepsilon_{b, \lambda_{N}}
\end{array}\right]\left[\begin{array}{c}
C_{H b}(\mathbf{x}) \\
C_{H b O_{2}}(\mathbf{x}) \\
C_{b}(\mathbf{x})
\end{array}\right]
$$

where $\varepsilon_{H b, \lambda_{1}}, \varepsilon_{H b, \lambda_{1}}$, and $\varepsilon_{H b, \lambda_{1}}$, and $C_{\mathrm{HbO}_{2}}(\mathbf{x}), C_{\mathrm{HbO}_{2}}(\mathbf{x})$, and $C_{\mathrm{HbO}_{2}}(\mathbf{x})$ are the molar extinction coefficients and concentrations of deoxy-hemoglobin, oxy-hemoglobin, and the blue product, respectively. We are interested in estimating the concentration distributions, but this is complicated by wavelength- and spatial dependencies of optical fluences in tissue. To make further progress, approximations are required. We assume that the fluence distributions can be written as: 
$\Phi_{j}(\mathbf{x}) \approx \varphi_{j}(\mathbf{r}) \Phi(\mathbf{x})+\Delta \Phi_{j}(\mathbf{x})$

where $\Phi(\mathbf{x})$ is a wavelength-independent fluence distribution and $\varphi_{j}(\mathbf{r})$ is a wavelength-dependent compensation factor, defined over image space, and such that $\varphi_{j}(\mathbf{r})$ is slowly varying compared to the point-spread function. $\Delta \Phi_{j}(\mathbf{x})$ is a perturbation term to make up for differences between the true fluence and the approximation $\varphi_{j}(\mathbf{r}) \Phi(\mathbf{x})$. With the approximation, the system of equations reduces to:

$\left[\begin{array}{c}p_{1}(\mathbf{r}) / \varphi_{1}(\mathbf{r}) \\ p_{2}(\mathbf{r}) / \varphi_{2}(\mathbf{r}) \\ \vdots \\ p_{N}(\mathbf{r}) / \varphi_{N}(\mathbf{r})\end{array}\right]=\left[\begin{array}{ccc}\varepsilon_{H b, \lambda_{1}} & \varepsilon_{H b O_{2}, \lambda_{1}} & \varepsilon_{b, \lambda_{1}} \\ \varepsilon_{H b, \lambda_{2}} & \varepsilon_{H b O_{2}, \lambda_{2}} & \varepsilon_{b, \lambda_{2}} \\ \vdots & \vdots & \vdots \\ \varepsilon_{H b, \lambda_{N}} & \varepsilon_{H b O_{2}, \lambda_{N}} & \varepsilon_{b, \lambda_{N}}\end{array}\right]\left[\begin{array}{c}\int h(\mathbf{x}, \mathbf{r}) \Phi(\mathbf{x}) C_{H b}(\mathbf{x}) d \mathbf{x} \\ \int h(\mathbf{x}, \mathbf{r}) \Phi(\mathbf{x}) C_{b}(\mathbf{x}) d \mathbf{x}\end{array}\right]\left[\begin{array}{c}b_{1} \\ b_{2} \\ \vdots \\ b_{N}\end{array}\right]$

Where the left-hand vector (which we'll label $\mathbf{p}_{\mathrm{o}}$ ) is a column of the compensated photoacoustic images, and the vector $\mathbf{c}=\left[\begin{array}{c}\int h(\mathbf{x}, \mathbf{r}) \Phi(\mathbf{x}) C_{H b}(\mathbf{x}) d \mathbf{x} \\ \int h(\mathbf{x}, \mathbf{r}) \Phi(\mathbf{x}) C_{H b O_{2}}(\mathbf{x}) d \mathbf{x} \\ \int h(\mathbf{x}, \mathbf{r}) \Phi(\mathbf{x}) C_{b}(\mathbf{x}) d \mathbf{x}\end{array}\right]$

contains as it's elements the images of concentration distributions assuming wavelength-independent fluence $\Phi(\mathbf{x})$. For example, $\Phi(\mathbf{x})$ could be chosen to be the fluence distribution for a particular wavelength. The vector $\mathbf{b}$ is the resulting bias term resulting from the approximation and it's elements are given as

$b_{j}(\mathbf{r})=\int h(\mathbf{x}, \mathbf{r}) \Delta \Phi_{j}(\mathbf{x}) \mu_{a, j}(\mathbf{x}) d \mathbf{x}$.

It is now possible to solve for the effective concentration vector $\mathbf{c}$ by linear least-squares matrix inversion

$\hat{\mathbf{c}}=\left(\boldsymbol{\varepsilon}^{T} \boldsymbol{\varepsilon}\right)^{-1} \boldsymbol{\varepsilon}^{T} \mathbf{p}_{0}$,

where a hat represents an estimated quantity and

$$
\boldsymbol{\varepsilon}=\left[\begin{array}{ccc}
\varepsilon_{H b, \lambda_{1}} & \varepsilon_{H b O_{2}, \lambda_{1}} & \varepsilon_{b, \lambda_{1}} \\
\varepsilon_{H b, \lambda_{2}} & \varepsilon_{H b O_{2}, \lambda_{2}} & \varepsilon_{b, \lambda_{2}} \\
\vdots & \vdots & \vdots \\
\varepsilon_{H b, \lambda_{N}} & \varepsilon_{H b O_{2}, \lambda_{N}} & \varepsilon_{b, \lambda_{N}}
\end{array}\right]
$$

is the matrix of molar extinction coefficients and $\left(\boldsymbol{\varepsilon}^{T} \boldsymbol{\varepsilon}\right)^{-1} \boldsymbol{\varepsilon}^{T}$ is the Moore-Penrose pseudo-inverse of the molar extinction matrix. The estimate $\hat{\mathbf{c}}$ differs from the true value $\mathbf{c}$ by the bias $\mathbf{c}_{b}=\left(\boldsymbol{\varepsilon}^{T} \boldsymbol{\varepsilon}\right)^{-1} \boldsymbol{\varepsilon}^{T} \mathbf{b}$. To accurately classify signals as originating from molecular agents, the bias term should be as low as possible to reduce false positives. One way to keep the element of $\mathbf{b}$ small is to use optical wavelengths spectrally close to each other, however, this may come at the cost of matrix inversion instability and noise amplification. 
The reconstructed images of the molecular imaging agent may not necessarily reflect the true, quantitative absorber distributions for a few reasons. First, it should be noted that the imaging system is sensitive to only a radial band of spatial frequencies as noted earlier. Second, for tomographic imaging systems, the reconstructed image is in a sense a projection of photoacoustic signals into the imaging plane and the extent and weighting of signals from different depths depends on the depth resolution of the imaging system, and the propagation of light. Third, and very important, if the absorption is very significant, all the incident light may be absorbed and the photoacoustic signal strength will be simply proportional to the incident laser pulse energy, and independent of the molar extinction spectra or concentration.

If we are merely interested in the non-quantitative spatial distribution of molecular agent signals, energy-dependent absorption of the agent ' $b$ ' is acceptable. Energy-dependent absorption of blood, however, is problematic for molecular imaging, since energy-dependent absorption of blood will bias energy into the reconstructed image of the molecular agent and provide possible false negatives. Maslov et al. ${ }^{7}$ provide an important discussion of these issues in the context of blood oxygenation estimation.

One way to help reduce bias is to use fine resolution - particularly depth resolution if the depth dimension has the greatest spatial changes in the fluence perturbations $\Delta \Phi_{j}(\mathbf{x})$. In this case, finer depth resolution will reduce the bias term, Eq. $\left({ }^{*}\right)$. Incidentally, modulation in the depth direction should also help reduce the bias terms - since modulation integrated over slow changes in the fluence perturbation will produce small values.

\subsection{Estimator Variance: Noise Propagation}

Random perturbations such as additive electronic noise will degrade system sensitivity to signals from molecular agents. It is important to understand how these perturbations propagate through the matrix inversion procedure. Understanding this propagation is important, for example, to help us select optical wavelengths for imaging. Without any assumptions about the probability distribution of the random perturbations except that the covariance of the noise process is matrix $\mathbf{K}_{\mathrm{n}}$, the variance of the molecular agent concentration estimates are given as:

$$
\sigma_{b}^{2}=\left\langle\Delta c_{b}^{2}\right\rangle=\left[\mathbf{K}_{\mathbf{c}}\right]_{33}
$$

where

$$
\mathbf{K}_{\mathbf{c}}=\left(\boldsymbol{\varepsilon}^{T} \boldsymbol{\varepsilon}\right)^{-1} \boldsymbol{\varepsilon}^{T} \mathbf{K}_{\mathbf{n}} \boldsymbol{\varepsilon}\left(\boldsymbol{\varepsilon}^{T} \boldsymbol{\varepsilon}\right)^{-1}
$$

is the covariance matrix of $\hat{\mathbf{c}}$. There is significant intuition to this if we consider a White Gaussian Noise (WGN) process with variance $\sigma^{2}$ such that $\mathbf{K}_{\mathbf{n}}=\sigma^{2} \mathbf{I}$. In this case (see Appendix)

$$
\sigma_{b}^{2}=\sigma^{2} \frac{1}{\left|\boldsymbol{\varepsilon}_{3}\right|^{2}\left(1-\rho^{2}\right)}
$$

Here $\left|\boldsymbol{\varepsilon}_{3}\right|$ is the norm of the column vector, where the column vectors for $\mathrm{Hb}, \mathrm{HbO}_{2}$ and $b$ are given as

$$
\boldsymbol{\varepsilon}_{1}=\left[\begin{array}{c}
\varepsilon_{H b, \lambda_{1}} \\
\varepsilon_{H b, \lambda_{2}} \\
\vdots \\
\varepsilon_{H b, \lambda_{N}}
\end{array}\right] \quad \boldsymbol{\varepsilon}_{2}=\left[\begin{array}{c}
\varepsilon_{\mathrm{HbO}_{2}, \lambda_{1}} \\
\varepsilon_{\mathrm{HbO}_{2}, \lambda_{2}} \\
\vdots \\
\varepsilon_{\mathrm{HbO}_{2}, \lambda_{N}}
\end{array}\right] \quad \boldsymbol{\varepsilon}_{3}=\left[\begin{array}{c}
\varepsilon_{b, \lambda_{1}} \\
\varepsilon_{b, \lambda_{2}} \\
\vdots \\
\varepsilon_{b, \lambda_{N}}
\end{array}\right]
$$

And $\rho$ is the spectral similarity factor, defined as

$$
\rho=\frac{\sqrt{\cos ^{2} \theta_{13}+\cos ^{2} \theta_{23}-2 \cos \theta_{13} \cos \theta_{23} \cos \theta_{12}}}{\sin \theta_{12}} .
$$

Here the angles $\theta_{\mathrm{ij}}$ are defined as the angles between the vectors $\varepsilon_{\mathrm{i}}$ and $\varepsilon_{\mathrm{j}}$. 
The relative signal strength of the molecular agent $b$ relative to the propagated noise is given as

$$
S N R_{\text {estimate }}=\frac{c_{b}}{\sigma_{b}}=\frac{c_{b}}{\sigma}\left|\boldsymbol{\varepsilon}_{3}\right| \sqrt{\left(1-\rho^{2}\right)} \approx \frac{\bar{\varepsilon}_{b} c_{b}}{\sigma} \sqrt{N\left(1-\rho^{2}\right)} .
$$

where $c_{b}$ is the concentration of the molecular agent ' $b$ ', $N$ is the number of optical wavelengths used, and $\bar{\varepsilon}_{b}$ is the mean molar extinction coefficient such that

$\left|\boldsymbol{\varepsilon}_{3}\right|^{2}=\varepsilon_{b, \lambda_{1}}^{2}+\varepsilon_{b, \lambda_{2}}^{2}+\cdots+\varepsilon_{b, \lambda_{N}}^{2} \approx N \bar{\varepsilon}_{b}^{2}$.

The geometric interpretation for the spectral similarity factor is the diameter of the unique circle (called a circumcircle) that intersects the triangle formed by the projection points of the unit vector $\hat{\boldsymbol{\varepsilon}}_{3}=\hat{\boldsymbol{\varepsilon}}_{b}$ onto $\hat{\boldsymbol{\varepsilon}}_{1}=\hat{\boldsymbol{\varepsilon}}_{\mathrm{Hb}}$ and $\hat{\boldsymbol{\varepsilon}}_{2}=\hat{\boldsymbol{\varepsilon}}_{\mathrm{HbO}_{2}}$, as shown in Fig. 3. When the molar extinction vectors are orthogonal the circumcircle shrinks to zero diameter and the $S N R$ is maximized. On the other hand, when the molar extinction vectors are parallel, the circumcircle is of unit diameter and the $S N R$ shrinks to zero. Rationally, the $S N R$ improves as the square root of the number of wavelengths used, but decreases as the spectral similarity factor increases.

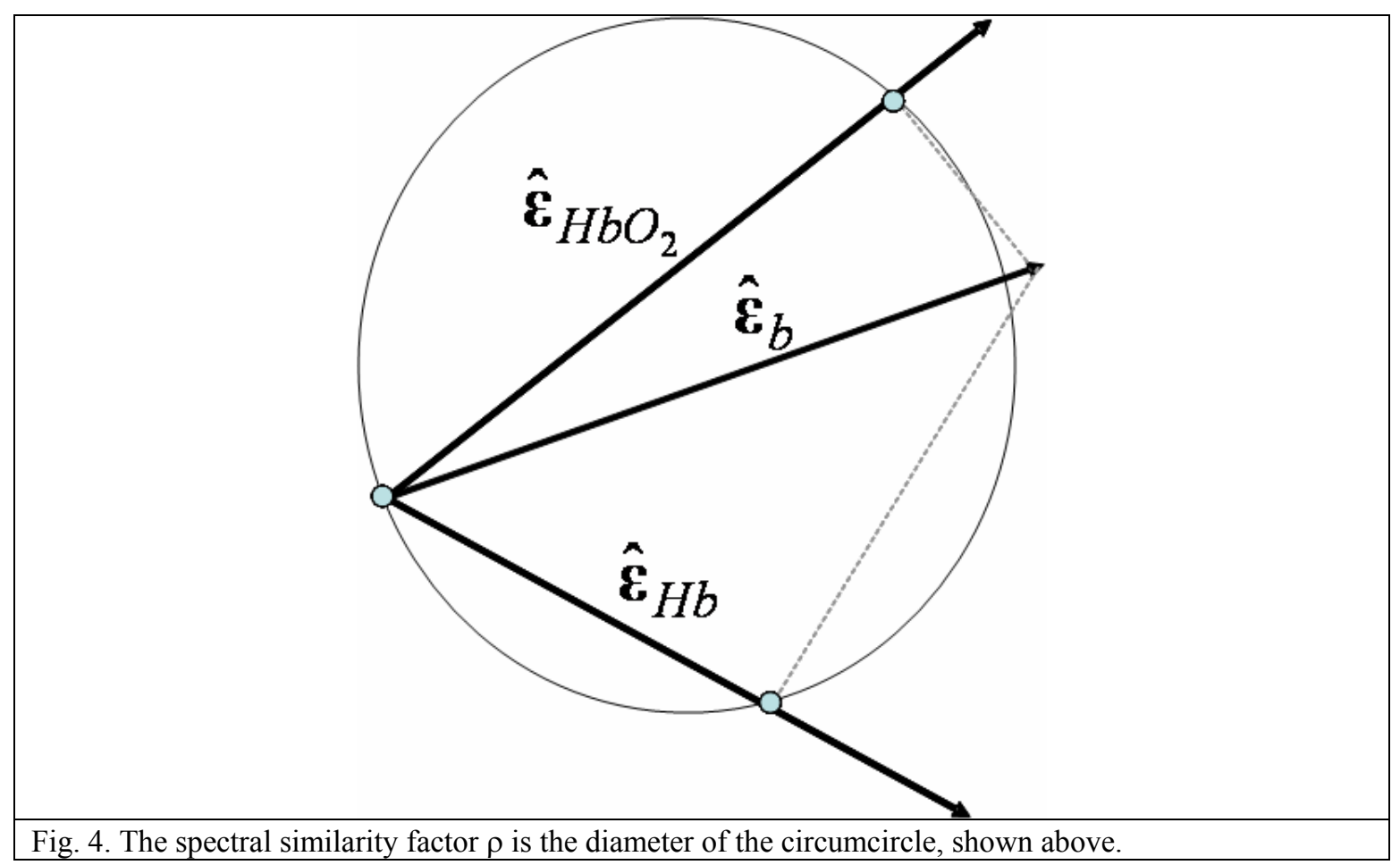

\subsection{Wavelength Selection}

Selection of the optical wavelengths to use for photoacoustic imaging is an important aspect of system design. If optical wavelengths are chosen to be spectrally distant, the spectral similarity factor may potentially be large if the spectra are very different. In this case $S N R$ may be maximal but the bias due to errors in fluence may be large. Conversely, wavelengths chosen spectrally near each other may have small bias but may amplify noise and other perturbations. These tradeoffs deserve careful consideration in designing systems. An additional practical limitation is the availability of widely tuneable laser sources. The spectral tuneability range will ultimately limit the spectral distances between wavelengths unless further laser source innovation emerges. 


\section{Conclusions}

The present work is a theoretical treatment of fundamental issues related to the performance of photoacoustic imaging systems for molecular imaging. We have shown that the system point-spread function for circular scanning photoacoustic tomography is related to the Hankel transform of the transducer frequency response, hence the system is sensitive to a radial band of spatial frequencies. We have also shown conditions for which matrix inversion can be performed and that estimator bias is generated whenever there are errors in fluence estimation. Improving depth resolution to less than the optical attenuation lengths in dominant absorbers may help reduce bias in molecular agent estimates, analogous to the discussion by Maslov et al. for estimation of quantitative blood oxygenation. If spatial localization, and not quantitative estimation is an acceptable goal then energy dependent absorption of the molecular tagging agent is tolerable, but energy-dependent absorption of blood is problematic. Estimator bias may introduce false positives for the task of detecting spatial regions of molecular signals in molecular imaging. Estimator variance, due to propagation of random perturbations such as additive electronic noise through the matrix inversion process will also impact molecular sensitivity. Simple noise propagation models have been developed to help aid system design in issues such as wavelength selection. A better understanding of these fundamental issues will be essential for future progress in photoacoustic molecular imaging.

\section{Acknowledgements}

We appreciate fruitful discussions with Dr. Konstantin Maslov. We gratefully acknowledge funding from the National Institutes of Health R01 NS46214 and R01 EB000712.

\section{Appendix}

If we consider a White Gaussian Noise (WGN) process with variance $\sigma^{2}$ such that $\mathbf{K}_{\mathbf{n}}=\sigma^{2} \mathbf{I}$. In this case,

$$
\sigma_{b}^{2}=\left[\mathbf{K}_{\mathbf{c}}\right]_{33}=\sigma^{2} \frac{\left|\begin{array}{ll}
\boldsymbol{\varepsilon}_{1}^{T} \boldsymbol{\varepsilon}_{1} & \boldsymbol{\varepsilon}_{1}^{T} \boldsymbol{\varepsilon}_{2} \\
\boldsymbol{\varepsilon}_{2}^{T} \boldsymbol{\varepsilon}_{1} & \boldsymbol{\varepsilon}_{2}^{T} \boldsymbol{\varepsilon}_{2}
\end{array}\right|}{\operatorname{det}\left(\boldsymbol{\varepsilon}^{T} \boldsymbol{\varepsilon}\right)}
$$

where

$$
\operatorname{det}\left(\boldsymbol{\varepsilon}^{T} \boldsymbol{\varepsilon}\right)=\left|\boldsymbol{\varepsilon}_{1}\right|^{2}\left|\boldsymbol{\varepsilon}_{2}\right|^{2}\left|\boldsymbol{\varepsilon}_{3}\right|^{2} \operatorname{det}\left(\begin{array}{ccc}
1 & \cos \theta_{12} & \cos \theta_{13} \\
\cos \theta_{12} & 1 & \cos \theta_{23} \\
\cos \theta_{13} & \cos \theta_{23} & 1
\end{array}\right)
$$

Then we have

$$
\begin{aligned}
& \sigma_{b}^{2}=\sigma^{2} \frac{\left|\boldsymbol{\varepsilon}_{1}\right|^{2}\left|\boldsymbol{\varepsilon}_{2}\right|^{2}\left(1-\cos ^{2} \theta_{12}\right)}{\left|\boldsymbol{\varepsilon}_{1}\right|^{2}\left|\boldsymbol{\varepsilon}_{2}\right|^{2}\left|\boldsymbol{\varepsilon}_{3}\right|^{2}\left(1+2 \cos \theta_{13} \cos \theta_{23} \cos \theta_{12}-\cos ^{2} \theta_{13}-\cos ^{2} \theta_{23}-\cos ^{2} \theta_{12}\right)} . \\
& =\sigma^{2} \frac{\sin ^{2} \theta_{12}}{\left|\boldsymbol{\varepsilon}_{3}\right|^{2}\left(\sin ^{2} \theta_{12}-d^{2}\right)}=\sigma^{2} \frac{1}{\left|\boldsymbol{\varepsilon}_{3}\right|^{2}\left(1-d^{2} / \sin ^{2} \theta_{12}\right)}
\end{aligned}
$$

where, by the law of cosines, $d$ is the distance between projection points:

$d^{2}=\cos ^{2} \theta_{13}+\cos ^{2} \theta_{23}-2 \cos \theta_{13} \cos \theta_{23} \cos \theta_{12}$

and, by the law of sines, $\rho$ is the diameter of the unique circumcircle

$\rho=d / \sin \theta_{12}$. 


\section{References}

${ }^{1}$ T. F. Massoud and S. S. Gambhir, "Molecular imaging in living subjects: seeing fundamental biological processes in a new light," Genes and Development, 17, 545-580.

${ }^{2}$ R. Weissleder, "Molecular imaging: exploring the next frontier," Radiology 212, pp. 609-614, 1999

${ }^{3}$ E. E. Graves, J. Ripoll, R. Weissleder, and V. Ntziachristos, "A sub-millimeter resolution fluorescence molecular imaging system for small animal imaging," Med. Phys. 30, pp. 901-911, 2003.

${ }^{4}$ P.R. Contag, I. N. Olomu, D.K. Stevenson, and C.H. Contag, "Bioluminescent indicators in living mammals," Nature Medicine 4, pp. 245-247, 1998

${ }^{5} \mathrm{M}$. Xu, Y. Yu, and L.-H. Wang, "Time-domain reconstruction algorithms and numerical simulations for thermoacoustic tomography in various geometries." IEEE Trans. Biomed. Eng. 50 (9), pp. 1086-1099, 2003.

${ }^{6}$ X. Xie, M. L Li, J. T. Oh, G. Ku, W. Wang, C. Li, S. Similache, G.F. Lungu, G. Stoica, and L.-H. Wang, "Photoacoustic molecular imaging of small animals in vivo", Proc. SPIE 2006.

${ }^{7}$ K. Maslov, M. Sivaramakrishnan, H. Zhang, G. Stoica, L.-H. Wang, "Technical considerations in Quantitative Blood Oxygenation Measurement Using Photoacoustic Microscopy in vivo", Proc. SPIE, 2006.

${ }^{8}$ L. Li, R.J. Zemp, G.F. Lungu, G. Stoica, and L.-H. Wang, "Photoacoustic Imaging of Gene Expression in vivo", Proc. SPIE, 2006 